\section{Consumo regular de frutas e hortaliças por estudantes universitários em Rio Branco, Acre, Brasil: prevalência e fatores associados}

\author{
Regular consumption of fruits and vegetables \\ by university students in Rio Branco, Acre State, \\ Brazil: prevalence and associated factors
}

\author{
1 Programa de Pós-graduação \\ em Saúde Coletiva, \\ Universidade Federal do Acre, \\ Rio Branco, Brasil. \\ 2 Centro de Ciências da \\ Saúde e do Desporto, \\ Universidade Federal do Acre, \\ Rio Branco, Brasil. \\ Correspondência \\ A. A. Ramalho \\ Programa de Pós-graduação \\ em Saúde Coletiva, \\ Universidade Federal do Acre. \\ Campus Universitário, $B R$ \\ 364, Km 04, Rio Branco, AC \\ 69915-900, Brasil \\ alandersonalves@hotmail.com
}

\section{Abstract}

This cross-sectional study with university students examined the prevalence of regular consumption of fruits and vegetables and associated factors among students at a Federal university in Rio Branco, Acre State, Brazil. 863 undergraduates were interviewed in 2010. Overall prevalence of regular consumption of fruits and vegetables was 14.8\%. Factors associated with regular consumption of fruits and vegetables were: socioeconomic classes $A$ and $B$ (PR = 1.70; 95\%CI: 1.10-2.62), living with a partner ( $P R=1.53$; 95\%CI: 1.02-2.29), regular physical activity ( $P R=1.69 ; 95 \% C I$ : 1.112.56), and consumption of fast food twice a week or less (PR =1.49; 95\%CI: 1.04-2.13). A minority of the students met the recommendation to consume fruits and vegetables 5 or more days a week. Regular fruit and vegetable consumption was associated with socioeconomic status and healthy habits such as physical exercise and low consumption offast food.

Fruits; Vegetables; Food Consumption; Food Habits; Students
Alanderson Alves Ramalho 1
Tatiane Dalamaria 2
Orivaldo Florencio de Souza ${ }^{2}$

\section{Introdução}

A promoção do consumo de frutas e hortaliças é uma prioridade mundial para a melhoria da saúde da população 1 . Várias investigações evidenciaram o efeito protetor das frutas e hortaliças para doenças crônicas não transmissíveis ${ }^{2,3}$ e na manutenção do peso corporal ${ }^{4,5}$. Nesse sentido, a Organização Mundial da Saúde (OMS) ${ }^{6}$ recomenda o consumo diário de $400 \mathrm{~g}$ de frutas para a prevenção de doenças crônicas não transmissíveis. Em pesquisas populacionais, Block et al. 7 também recomendam verificar o consumo regular de frutas e hortaliças no mínimo 5 dias na semana como marcador de alimentação saudável. Essa última recomendação tem sido utilizada pelo Ministério da Saúde 8 na Vigilância de Fatores de Risco e Proteção para Doenças Crônicas por Inquérito Telefônico (VIGITEL) para o monitoramento da saúde da população brasileira.

Em 2003, Jaime \& Monteiro ${ }^{9}$ averiguaram na população adulta brasileira inadequação no consumo diário de frutas e hortaliças em 70\% e 59\%, respectivamente. Do mesmo modo, informações recentes da VIGITEL mostraram baixa prevalência de consumo regular de frutas e hortaliças na população brasileira ${ }^{8}$. As maiores disparidades ocorreram entre as capitais dos estados das regiões Norte e Nordeste em contraste com as capitais de estados das regiões Sul e Sudeste. Belém, Macapá e Rio Branco mostraram os mais 
baixos consumos regulares de frutas e hortaliças do Brasil. Em conformidade com os dados da VIGITEL, a Pesquisa de Orçamentos Familiares de 2008-2009 (POF) revelou que a disponibilidade domiciliar de frutas e hortaliças em Rio Branco também estava abaixo da média nacional 10 .

Algumas investigações evidenciaram alteração nos hábitos alimentares de estudantes após o ingresso em curso de ensino superior 11,12,13. Infere-se que a adaptação ao contexto universitário e as novas redes sociais influenciam na modificação dos hábitos alimentares. Concomitantemente a essa situação, vários acadêmicos têm jornada dupla: estudo e trabalho. Em consequência das longas distâncias ou curto período de tempo para o deslocamento entre o local de trabalho, o domicílio e a universidade, de fato alguns acadêmicos preferem alimentar-se em lanchonetes ou restaurantes. Além disso, vários estudantes após ingressarem no curso universitário necessitam mudar do município de residência dos pais, logo se tornando responsáveis pela própria alimentação. Essas situações corroboram a alta prevalência de alimentação não saudável e baixo relato de consumo de frutas e hortaliças observados em pesquisas com universitários brasileiros 11,12,13.

O consumo de frutas e hortaliças por estudantes universitários tem sido investigado no Brasil e em outros países 13,14,15,16,17. No entanto, não foram identificados trabalhos sobre os hábitos de consumo desses alimentos por estudantes universitários de Rio Branco. Assim, o objetivo desta investigação foi analisar a prevalência e os fatores associados ao consumo regular de frutas e hortaliças em acadêmicos de uma universidade pública federal no Município de Rio Branco, Estado do Acre.

\section{Método}

Trata-se de um estudo transversal com universitários, realizado entre agosto e novembro de 2010. A população da pesquisa era de aproximadamente 4.500 acadêmicos matriculados em 34 cursos de graduação na Universidade Federal do Acre, campus de Rio Branco. Para a determinação do tamanho mínimo da amostra foi adotada a prevalência esperada de $50 \%$, com a precisão fixada para um erro de amostragem em 0,05, nível de $95 \%$ de confiança e efeito de delineamento de 2. O tamanho mínimo estimado da amostra foi de 768 acadêmicos. Para proteger dos efeitos da não resposta, sendo considerado em 10\%, o tamanho final da amostra foi estimado em 845 estudantes. Na seleção dos acadêmicos foi utilizada amostragem por conglomerados em dois estágios para proporções desiguais, de acordo com os procedimentos sugeridos por Kish 18 e Kalton 19. As unidades primárias foram os cursos e as unidades secundárias os períodos dos cursos. De modo representativo da universidade, todos os cursos e os respectivos períodos foram considerados nos sorteios para a determinação da amostra do estudo. O critério de inclusão no trabalho foi estar regularmente matriculado e frequentando as aulas em curso de graduação sorteado na Universidade Federal do Acre, campus de Rio Branco.

Este projeto foi submetido e aprovado pelo Comitê de Ética em Pesquisa da Universidade Federal do Acre (processo: 23107.011544/2010-94).

A coleta dos dados foi realizada por questionário estruturado com 30 perguntas referentes às características demográficas, sociofamiliares, hábitos de vida e alimentares. O questionário foi pré-testado e aplicado por professores e pós-graduandos da área de ciências da saúde.

As variáveis independentes foram compostas por aspectos socioeconômicos, comportamentais e consumo alimentar. As variáveis demográficas e socioeconômicas foram sexo, idade, classe econômica, situação ocupacional, estado marital, número de filhos. As faixas etárias em anos foram categorizadas em igual ou menor de 20 anos, entre 21 e 30 e igual ou acima de 31 anos. A classe econômica foi definida pelos critérios da Associação Brasileira de Empresas de Pesquisa (Critérios de Classificação Econômica do Brasil. http://www.abep.org/novo/Content. aspx?ContentID-301, acessado em 09/Mai/2011) de 2011 em classes A, B, C, D e E. Para análise dos dados, esses critérios foram agrupados em classes alta (A e B) e baixa (C, D e E). Na variável situação ocupacional foram consideradas as respostas não trabalha e trabalha (todas as atividades remuneradas, com exceção das bolsas recebidas por atividades desenvolvidas em programas de ensino, pesquisa e extensão realizadas na universidade). A variável estado marital foi constituída pelas categorias sem companheiro (solteiros, separados ou viúvos) e com companheiro (casado ou em união consensual). Enquanto que o número de filhos foi categorizado em: nenhum e 1 ou mais filhos.

As variáveis comportamentais foram atividade física e local de refeições. A variável atividade física foi verificada mediante questões sobre a frequência semanal e duração por sessão das atividades de lazer e locomoção (caminhada e uso de bicicleta). A intensidade das atividades físicas relatadas foi determinada conforme recomendação de Ainsworth et al. 20. Posteriormente, foi realizada a classificação da atividade física conforme recomendações da Haskell et al. 21 em 
ativo (realizar atividade física de intensidade moderada com frequência semanal mínima de três vezes e duração diária de pelo menos 30 minutos ou atividade física de intensidade vigorosa com frequência semanal mínima de cinco vezes e duração diária de pelo menos 20 minutos) e sedentário (não atingir nenhum critério da categoria ativo). Também foi indagado aos acadêmicos sobre os locais das refeições principais. As respostas foram categorizadas em: somente em casa ou o combinado de em casa, lanchonetes ou restaurantes.

O consumo alimentar foi identificado por questões sobre a frequência semanal de ingestão de fast food (salgados, sanduíches e batatas fritas) e de doces, guloseimas e compotas. Para análise dos dados, ambas as variáveis foram categorizadas em: consumo em 3 ou mais dias na semana e 2 ou menos dias na semana.

A variável dependente consumo regular de frutas e hortaliças foi constituída mediante as questões sobre a frequência semanal do consumo desses nutrientes. Na questão das hortaliças foram desconsiderados a batata, a macaxeira e o inhame. Com base nas respostas foram determinados dois indicadores: consumo de frutas e consumo de hortaliças, ambas categorizadas em: regular (5 ou mais dias na semana) e irregular (4 ou menos dias na semana). Por fim, a variável dependente foi elaborada baseando-se na combinação dos dois indicadores iniciais, sendo: consumo regular de frutas e hortaliças em 5 ou mais dias na semana e o consumo irregular em 4 ou menos dias na semana.

Os dados foram digitados seguindo a rotina elaborada no programa EpiData (Epidata Association, Odense, Dinamarca). Após o processo de digitação, os dados foram transportados para o programa estatístico Stata 9.2 (Stata Corp., College Station, Estados Unidos), no qual foram realizadas as categorizações e as análises estatísticas. Todas as análises foram realizadas no módulo svy.

As prevalências do consumo regular de frutas, consumo regular de hortaliças e consumo regular de frutas e hotaliças com os respectivos intervalos de 95\% de confiança (IC95\%) foram calculados para amostra total e por sexo. O teste qui-quadrado foi utilizado para identificar as associações entre os sexos ( $\mathrm{p}<0,05)$.

Os fatores associados ao consumo regular de frutas e hortaliças foram analisados em duas etapas. Inicialmente, as variáveis independentes que apresentaram associações com consumo regular de frutas e hortaliças com valor de $\mathrm{p}<0,20$ (teste de qui-quadrado para heterogeneidade e de tendência linear) foram selecionadas para compor o modelo múltiplo final.
Posteriormente, foram identificados os fatores associados ao consumo regular de frutas e hortaliças mediante análise múltipla e hierarquizada de regressão de Poisson 22,23. Utilizou-se o modelo conceitual adaptado de Figueiredo et al. 24 (Figura 1). As variáveis sexo e faixa etária foram inseridas no primeiro bloco e permaneceram nas etapas subsequentes. Em cada bloco foram introduzidas todas as variáveis selecionadas na primeira etapa. Todas as variáveis que apresentaram valor de $\mathrm{p}<0,05$ foram selecionadas como fator associado ao consumo regular de

Figura 1

Modelo conceitual hierarquizado para análise do consumo regular de frutas e hortaliças por estudantes universitários de Rio Branco, Acre, Brasil, 2010.

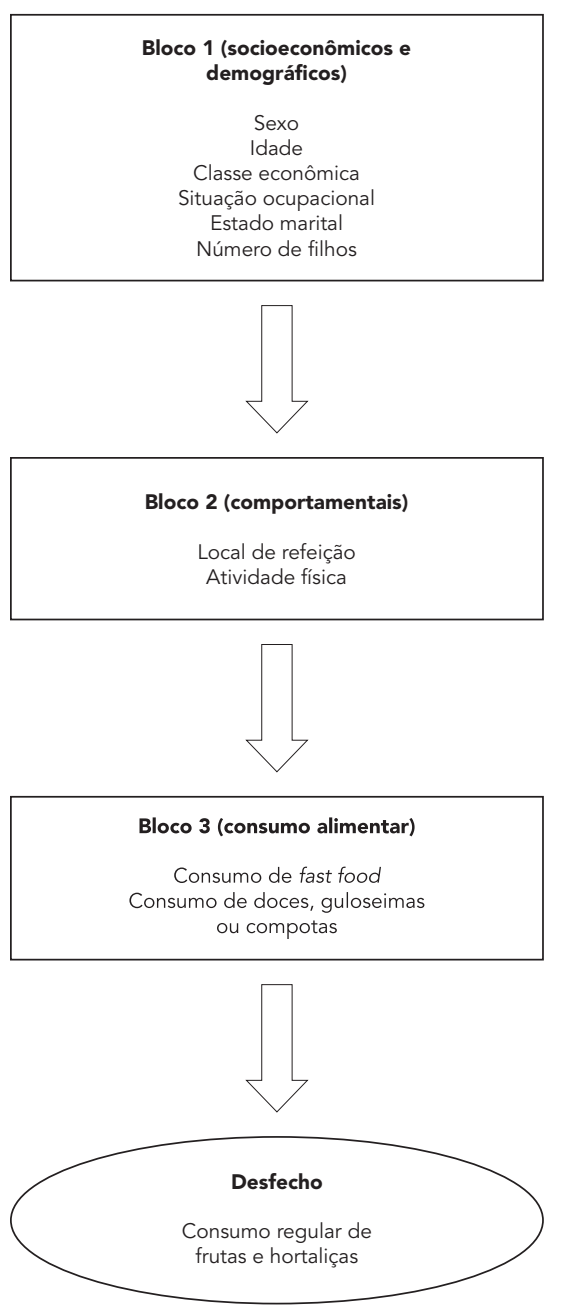

Fonte: adaptado de Figueiredo et al. 24. 
frutas e hortaliças e permaneceram nos modelos múltiplos nas análises dos blocos subsequentes. As variáveis que apresentaram valor de $\mathrm{p} \geq$ 0,05 foram retiradas do modelo e avaliadas para verificar se havia alterações em mais de $10 \%$ na magnitude das razões de prevalência das variáveis que permaneceram no modelo. Nos casos de ocorrência de alterações na magnitude da razão de prevalência acima de $10 \%$ nas variáveis que permaneceram no modelo, a variável foi reinserida e mantida nos modelos múltiplos dos blocos subsequentes. O poder do estudo a posteriori por bloco mediante regressão de Poisson foi calculado com o auxílio do programa G*Power 3.1.3 (http:// www.psycho.uni-duesseldorf.de/abtei lungen/aap/gpower3).

\section{Resultados}

Dos 893 acadêmicos contatados, houve 19 recusas na participação do estudo e 11 foram excluídos por não responderem adequadamente às questões referentes ao consumo de frutas, legumes e verduras contidas no questionário. Dos 863 estudantes participantes, $38,2 \%$ eram homens e $61,8 \%$ mulheres. Desses, $37,1 \%$ tinham idades iguais ou menores de 20 anos, $44,8 \%$ com idades entre 21 e 30 anos, e 18,1\% com idades iguais ou acima de 31 anos.

A prevalência geral de consumo regular de frutas e hortaliças foi de $14,8 \%$ (Tabela 1). Na amostra geral e estratificada por sexo observouse que os acadêmicos apresentavam aproximadamente o dobro do consumo de hortaliças em 5 ou mais dias na semana em relação às frutas. Entretanto, não foi identificada diferença estatisticamente significante entre os sexos no consumo regular combinado de frutas e hortaliças.
Com base na Tabela 2 verificou-se que as faixas etárias maiores apresentavam maior consumo regular de frutas e hortaliças (p para tendência linear $=0,004$ ). Neste estudo, não foram identificados estudantes na classe econômica E. As variáveis explanatórias selecionadas como candidatas para compor o modelo final foram: classe econômica, estado marital, número de filhos, atividade física, local de refeições e consumo de fast food.

Na Tabela 3 estão apresentados os fatores associados ao consumo regular de frutas e hortaliças. Nenhuma variável eliminada alterou em mais de $10 \%$ a magnitude das razões de prevalências das variáveis que permaneceram no modelo em cada bloco. Os acadêmicos pertencentes às classes econômicas A e B mostraram 1,70 vez mais probabilidade de consumir regularmente frutas e hortaliças em relação aos acadêmicos das classes C e D. A situação de conviver com companheiro mostrou 1,53 vez mais probabilidade de consumo regular de frutas e hortaliças em contraste aos estudantes que viviam sem companheiro. Nos comportamentos saudáveis, ser fisicamente ativo e o hábito de consumir fast food duas ou menos vezes na semana mostraram magnitudes de associação de 1,69 e 1,49, respectivamente, com o consumo de frutas e hortaliças em 5 ou mais dias na semana.

Foi calculado o poder estatístico a posteriori relativo às estimativas das razões de prevalências ajustadas mediante regressão de Poisson. Verificou-se no primeiro e no segundo blocos o poder estatístico de $80 \%$ para calcular como estatisticamente significativa as razões de prevalência igual ou superior a 1,22 . No terceiro bloco, também ao poder estatístico de $80 \%$, foi possível detectar como estatisticamente significante a razão de prevalência igual ou superior a 1,21.

Distribuição da frequência de 5 dias ou mais no consumo de frutas, hortaliças e ambas combinadas, segundo sexo de estudantes universitários de Rio Branco, Acre, Brasil, 2010.

\begin{tabular}{|c|c|c|c|c|c|c|c|}
\hline & \multicolumn{2}{|c|}{$\begin{array}{c}\text { Masculino } \\
(n=330)\end{array}$} & \multicolumn{2}{|c|}{$\begin{array}{l}\text { Feminino } \\
(n=533)\end{array}$} & \multirow[t]{2}{*}{ Valor de $p$} & \multicolumn{2}{|c|}{$\begin{array}{c}\text { Todos } \\
(\mathrm{n}=863)\end{array}$} \\
\hline & $\%$ & IC95\% & $\%$ & IC95\% & & $\%$ & IC95\% \\
\hline Consumo regular de frutas & 18,5 & $13,5-24,8$ & 21,4 & $16,8-26,9$ & 0,305 & 20,3 & $16,2-25,4$ \\
\hline Consumo regular de hortaliças & 32,7 & $26,7-39,2$ & 44,6 & $39,2-50,1$ & 0,014 & 40,0 & $35,7-44,5$ \\
\hline Consumo regular de frutas e hortaliças & 12,4 & $7,7-19,3$ & 16,3 & $12,2-21,4$ & 0,256 & 14,8 & $11,3-19,2$ \\
\hline
\end{tabular}

IC95\%: intervalo de 95\% de confiança. 
Prevalência e razão de prevalência do consumo regular de frutas e hortaliças segundo características demográficas, socioeconômicas, comportamentais e consumo alimentar de estudantes universitários de Rio Branco, Acre, Brasil, 2010.

\begin{tabular}{|c|c|c|c|c|c|}
\hline & $n$ * & Prevalência & $\mathrm{RP}$ & IC95\% & Valor de $p$ \\
\hline \multicolumn{6}{|l|}{ Sexo } \\
\hline Masculino & 330 & 12,4 & 1,00 & & \\
\hline Feminino & 533 & 16,3 & 1,31 & $0,79-2,15$ & 0,262 \\
\hline \multicolumn{6}{|l|}{ Idade (anos) } \\
\hline$\leq 20$ & 306 & 11,4 & 1,00 & & \\
\hline $21-30$ & 369 & 14,6 & 1,27 & $0,76-2,13$ & 0,326 \\
\hline$\geq 31$ & 149 & 21,4 & 1,87 & $1,05-3,33$ & 0,033 \\
\hline p tendência linear & & & & & 0,004 \\
\hline \multicolumn{6}{|l|}{ Classe econômica } \\
\hline$C$ e D & 470 & 11,0 & 1,00 & & \\
\hline$A$ e $B$ & 387 & 19,3 & 1,75 & $1,19-2,56$ & 0,006 \\
\hline \multicolumn{6}{|l|}{ Situação ocupacional } \\
\hline Não trabalha & 539 & 13,9 & 1,00 & & \\
\hline Trabalha & 321 & 16,5 & 1,18 & $0,88-1,58$ & 0,232 \\
\hline \multicolumn{6}{|l|}{ Estado marital } \\
\hline Sem companheiro & 631 & 12,2 & 1,00 & & \\
\hline Com companheiro & 228 & 21,9 & 1,79 & $1,21-2,66$ & 0,006 \\
\hline \multicolumn{6}{|l|}{ Número de filhos } \\
\hline Nenhum & 615 & 12,8 & 1,00 & & \\
\hline 1 ou mais & 242 & 19,8 & 1,54 & $1,01-2,35$ & 0,044 \\
\hline \multicolumn{6}{|l|}{ Atividade física } \\
\hline Sedentário & 593 & 12,8 & 1,00 & & \\
\hline Ativo & 266 & 19,5 & 1,52 & $1,01-2,28$ & 0,048 \\
\hline \multicolumn{6}{|l|}{ Local de refeições } \\
\hline Em casa, lanchonetes ou restaurantes & 557 & 12,7 & 1,00 & & \\
\hline Somente em casa & 304 & 18,7 & 1,47 & $0,94-2,29$ & 0,084 \\
\hline \multicolumn{6}{|l|}{ Consumo de fast food (dias da semana) } \\
\hline 3 ou mais & 480 & 11,2 & 1,00 & & \\
\hline 2 ou menos & 382 & 19,3 & 1,72 & $1,15-2,56$ & 0,011 \\
\hline \multicolumn{6}{|l|}{ Consumo de doces, guloseimas ou compotas } \\
\hline \multicolumn{6}{|l|}{ (dias da semana) } \\
\hline 3 ou mais & 448 & 14,0 & 1,00 & & \\
\hline 2 ou menos & 415 & 15,6 & 1,11 & $0,88-1,39$ & 0,327 \\
\hline
\end{tabular}

IC95\%: intervalo de 95\% de confiança; RP: razão de prevalência.

* Missings existentes em algumas variáveis por não resposta.

\section{Discussão}

Baixa prevalência de consumo regular de frutas e hortaliças de 14,8\% foi evidenciada em acadêmicos da Universidade Federal do Acre, campus de Rio Branco. Os maiores consumos foram evidenciados nas faixas etárias mais altas e no sexo feminino. Os fatores contribuintes para o consumo regular de frutas e hortaliças pelos estudantes foram: pertencer à classe econômica $\mathrm{A}$ ou $\mathrm{B}$, conviver com companheiro e ter hábitos saudáveis relacionados à prática de atividade física e consumo de fast food igual ou menor que 2 vezes na semana.

$\mathrm{O}$ alto consumo regular de frutas e hortaliças nas faixas etárias maiores verificado em acadêmicos do presente trabalho concorda com resultados anteriores da Pesquisa Mundial de Saúde 9 e outros estudos nacionais 24,25,26. Conforme explicação de Jaime \& Monteiro 9 e Jaime et al. 27 para o mesmo fenômeno em população adulta brasileira, sugere-se que os acadêmicos com 
Fatores associados ao consumo regular de frutas e hortaliças por estudantes universitários de Rio Branco, Acre, Brasil, 2010.

\begin{tabular}{|c|c|c|c|}
\hline & RP ajustada & IC95\% & Valor de $p$ \\
\hline \multicolumn{4}{|l|}{ Classe econômica * } \\
\hline C e D & 1,00 & & \\
\hline$A$ e $B$ & 1,70 & $1,10-2,62$ & 0,018 \\
\hline \multicolumn{4}{|l|}{ Estado marital * } \\
\hline Sem companheiro & 1,00 & & \\
\hline Com companheiro & 1,53 & $1,02-2,29$ & 0,037 \\
\hline \multicolumn{4}{|l|}{ Atividade física ** } \\
\hline Sedentário & 1,00 & & \\
\hline Ativo & 1,69 & $1,11-2,56$ & 0,016 \\
\hline \multicolumn{4}{|c|}{ Consumo de fast food ${ }^{\star \star \star}$ (dias da semana) } \\
\hline 3 ou mais & 1,00 & & \\
\hline 2 ou menos & 1,49 & $1,04-2,13$ & 0,030 \\
\hline \multicolumn{4}{|c|}{ IC95\%: intervalo de 95\% de confiança; RP: razão de prevalência. } \\
\hline \multicolumn{4}{|c|}{ * Ajustado para sexo, idade; } \\
\hline \multicolumn{4}{|c|}{ ** Ajustado sexo, idade, classe econômica e estado marital; } \\
\hline
\end{tabular}

idades maiores podem apresentar melhor cuidado com a saúde. Entretanto, o efeito coorte na formação dos hábitos alimentares também citado por Jaime \& Monteiro ${ }^{9}$ e Jaime et al. 27 pode ser uma explicação improvável para esses estudantes universitários, pois a maioria $(81,9 \%)$ tinha idade inferior a 30 anos.

Entre todas as capitais brasileiras, os dados do VIGITEL revelaram que a população adulta de Rio Branco apresentou a terceira menor prevalência $(20,9 \%)$ de consumo regular de frutas e hortaliças 8 . De modo alarmante, no grupo específico dos estudantes universitários nas faixas etárias abaixo de 30 anos observou-se magnitude inferior de prevalência de consumo regular de frutas e hortaliças. Apenas a faixa etária igual ou acima de 30 anos mostrou magnitude aproximada à prevalência geral de Rio Branco.

Do mesmo modo, o baixo consumo regular de frutas e hortaliças dos acadêmicos de Rio Branco contrapõe as informações de consumo desses alimentos por universitários de outras instituições de ensino superior e de escolares do ensino fundamental do Brasil. Nesse sentido, o consumo regular de frutas e hortaliças pelos estudantes de nível superior de Rio Branco mostrou-se abaixo da prevalência de 24,9\% evidenciada em acadêmicos da área de saúde de Brasília 13. Em outra universidade pública brasileira foi verificado consumo regular de frutas em $25 \%$ e de hortaliças em $72 \% 15$, sendo esses valores superiores aos observados nos universitários de Rio Branco.
Essas diferenças mantiveram-se quando comparadas com estudantes do nono ano do ensino fundamental participantes da Pesquisa Nacional de Saúde do Escolar 28 de 2009, em que a prevalência evidenciada de consumo regular de frutas foi de $31,5 \%$ e de hortaliças de $31,4 \%$.

Dados da POF de 2008-2009 10 revelaram que no Município de Rio Branco havia a terceira menor disponibilidade domiciliar de frutas e sucos naturais, e a quarta menor de verduras e legumes em comparação às outras capitais brasileiras. Além disso, com relação à cesta básica nacional, dados do Departamento Intersindical de Estatística e Estudos Sócio-Econômicos (DIEESE. Bando de dados da Cesta Básica Nacional. http://turandot.dieese.org.br/bdcesta/ cesta.html, acessado em 29/Jul/2011) revelaram que em todos os meses de 2010 a banana e o tomate apresentaram preços médios superiores nas capitais investigadas da Região Norte, em relação às capitais da Região Sudeste. Desse modo, infere-se que os preços elevados de frutas e hortaliças observados na Região Norte dificultam a compra desses alimentos. Além disso, sugere-se que a baixa exposição domiciliar às frutas e hortaliças vivida pelos acadêmicos pode explicar, em parte, o baixo consumo desses alimentos. Por conseguinte, conclui-se que essas situações sejam responsáveis pelas diferenças no consumo regular de frutas e hortaliças entre universitários de Rio Branco e estudantes de outras instituições de Ensino Superior do Brasil 13,15. 
Devido ao alto custo das frutas e hortaliças, estudos nacionais e internacionais sugerem que a situação econômica é decisiva para a compra desses alimentos 29,30. Corroborando essa afirmativa, mediante dados da POF 2002-2003, Claro \& Monteiro 30 descreveram que o aumento de $1 \%$ na renda familiar per capita provocaria aumento de $0,27 \%$ na disponibilidade domiciliar de frutas e hortaliças. De acordo com esse contexto, os acadêmicos de Rio Branco com alto poder aquisitivo apresentaram maior consumo regular de frutas e hortaliças em relação aos das classes econômicas C e D. Apesar da maior renda não assegurar alimentação saudável, essa possibilita aos estudantes comporem uma dieta contendo frutas e hortaliças.

De acordo com estudo de revisão sistemática, Kamphuis et al. 31 evidenciaram que o hábito de consumir frutas e hortaliças mostrava-se maior entre os casados em contraste com os solteiros. Circunstância semelhante foi identificada entre os universitários de Rio Branco. Assim, sugerese que a situação dos acadêmicos conviverem com companheiro(a) representa um importante apoio social ao consumo regular de frutas e hortaliças. Além disso, infere-se que o hábito de alimentação saudável por um dos companheiros pode influenciar positivamente na maior disponibilidade desses alimentos no contexto domiciliar.

Coincidente aos resultados evidenciados no presente estudo, investigações internacionais 17,32 e nacionais 24,26 identificaram associação inversa entre o sedentarismo e o consumo regular de frutas e hortaliças. Essa associação confirma achados anteriores de que o hábito de consumir regularmente frutas e hortaliças coexiste com outros comportamentos saudáveis, como a prática regular de atividade física 33,34. Assim, de acordo com a OMS 1, sugere-se que tanto o hábito de consumir frutas e hortaliças em 5 ou mais dias na semana como a prática regular de atividade física são importantes preditores de comportamento saudável em acadêmicos de Rio Branco, indicando menor risco de doenças crônicas não transmissíveis.

No presente trabalho, o baixo consumo semanal de alimentos fast food mostrou associação com o consumo regular de frutas e hortaliças. Informação análoga foi verificada em indivíduos adultos no Município de São Paulo 24, em adolescentes dos Estados Unidos 35 e estudantes universitários da Grécia 12. Esses achados são consistentes com a tendência mundial de alterações de hábitos alimentares 36 . No Brasil, Levy-Costa et al. 37 identificaram aumento no consumo de alimentos com alto teor de gorduras e alimentos industrializados em contraste com a manutenção do insuficiente consumo de frutas e hortaliças.

Destaca-se que a generalização das informações evidenciadas é circunscrita à Universidade Federal do Acre no campus de Rio Branco. Algumas limitações deste estudo devem ser ressaltadas. Sugerimos cautela na comparação entre estudos pelo fato de haver diferenças nos métodos de definição e categorização de frutas e hortaliças. Concluímos que seja mínima a ocorrência do viés de memória porque as questões coletadas são eventos recentes. O consumo de frutas e hortaliças é um hábito desejável, logo pode haver superestimação no relato das respostas de consumo desses alimentos 38 . Entretanto, pela baixa prevalência identificada consideramos que esse tipo de erro de aferição tenha sido mínimo. Além disso, o delineamento transversal utilizado no presente trabalho impossibilitou a identificação da relação causal entre as variáveis independentes e o consumo regular de frutas e hortaliças.

Em situação diferente da população do sul e sudeste do Brasil, sugere-se que o elevado preço 26 e a baixa disponibilidade domiciliar 10 dificultam o acesso às frutas e hortaliças pelos estudantes universitários de Rio Branco. $\mathrm{O}$ acesso adequado a esses alimentos é importante para a saúde humana por serem ricos em micronutrientes e terem baixa densidade energética. Além disso, são alimentos protetores para várias doenças crônicas não transmissíveis 2,3,4,5. Considerando que os hábitos alimentares adotados durante a realização do curso de graduação podem perdurar nos anos posteriores e impactar sobre a qualidade da saúde, é necessário que os universitários de Rio Branco tenham acesso às frutas e hortaliças.

Em conclusão, foi evidenciado que somente uma minoria de acadêmicos alcançou a recomendação de consumir frutas e hortaliças em 5 ou mais dias da semana. Os universitários que viviam sem companheiro(a) e das classes econômicas C ou D mostraram-se mais suscetíveis ao consumo irregular de frutas e hortaliças. Os hábitos de vida saudáveis representados pela prática da atividade física e pelo baixo consumo semanal de fast food, apresentaram associações com o consumo regular de frutas e hortaliças entre acadêmicos de uma universidade pública federal do Município de Rio Branco. 


\section{Resumo}

Estudo transversal com universitários realizado para analisar a prevalência e os fatores associados ao consumo regular de frutas e hortaliças por acadêmicos de uma universidade pública federal de Rio Branco, Acre, Brasil. Foram investigados 863 estudantes de cursos de graduação em 2010. A prevalência geral de consumo regular de frutas e hortaliças foi de 14,8\%. Os fatores associados ao consumo regular de frutas e hortaliças foram: pertencer à classe econômica $A / B(R P=1,70$; IC95\%: 1,10-2,62), conviver com companheiro(a) $(R P=1,53$; IC95\%: 1,02-2,29), praticar atividade física ( $R P=1,69$; IC95\%: 1,11-2,56) e consumo de fast food igual ou menor que 2 vezes na semana ( $R P=1,49$; IC95\%: 1,04-2,13). Em conclusão, destaca-se que a minoria dos acadêmicos alcançou a recomendação de consumir frutas e hortaliças em 5 dias ou mais da semana. O consumo regular desses alimentos mostrou-se associado aos aspectos socioeconômicos e aos hábitos saudáveis relacionados à prática de atividade física e baixo consumo de fast food.

Frutas; Verduras; Consumo de Alimentos; Hábitos Alimentares; Estudantes

\section{Colaboradores}

A. A. Ramalho e T. Dalamaria participaram da coleta de dados, interpretação dos dados e redação do artigo. O. F. Souza contribuiu na elaboração do projeto, coleta de dados, interpretação dos dados e redação do artigo.

\section{Referências}

1. World Health Organization. Global strategy on diet, physical activity and health. Geneva: World Health Organization; 2004.

2. Pereira MA, O’Reilly E, Augustsson K, Fraser GE, Goldbourt U, Heitmann BL, et al. Dietary fiber and risk of coronary heart disease: a pooled analysis of cohort studies. Arch Intern Med 2004; 164:370-6.

3. Gao X, Chen H, Fung TT, Logroscino G, Schwarzschild MA, Hu FB, et al. Prospective study of dietary pattern and risk of Parkinson disease. Am J Clin Nutr 2007; 86:1486-94.

4. Rolls BJ, Ello-Martin JA, Tohill BC. What can intervention studies tell us about the relationship between fruit and vegetable consumption and weight management? Nutr Rev 2004; 62:1-17.

5. Sartorelli DS, Franco LJ, Cardoso MA. High intake of fruits and vegetables predicts weight loss in Brazilian overweight adults. Nutr Res 2008; 28:233-8.
6. World Health Organization. Diet, nutrition and the prevention of chronic diseases. Geneva: World Health Organization; 2003. (WHO Technical Report Series, 916).

7. Block G, Woods M, Potosky A, Clifford C. Validation of a self-administered diet history questionnaire using multiple diet records. J Clin Epidemiol 1990; 43:1327-35.

8. Secretaria de Vigilância em Saúde/Secretaria de Gestão Estratégica e Participativa, Ministério da Saúde. VIGITEL Brasil 2010: vigilância de fatores de risco e proteção para doenças crônicas por inquérito telefônico. Brasília: Ministério da Saúde; 2011.

9. Jaime PC, Monteiro CA. Fruit and vegetable intake by Brazilian adults, 2003. Cad Saúde Pública 2005; 21 Suppl 1:S19-24. 
10. Instituto Brasileiro de Geografia e Estatística. Pesquisa de Orçamentos Familiares - 2008-2009: análise nutricional da disponibilidade domiciliar de alimentos no Brasil. Rio de Janeiro: Instituto Brasileiro de Geografia e Estatística; 2010.

11. França C, Colares V. Estudo comparativo de condutas de saúde entre universitários no início e no final do curso. Rev Saúde Pública 2008; 42:420-7.

12. Papadaki A, Hondros G, Scott JA, Kapsokefalou M. Eating habits of university students living at, or away from home in Greece. Appetite 2007; 49:16976.

13. Marcondelli P, Costa THM, Schmitz BAS. Nível de atividade física e hábitos alimentares de universitários do 3o ao 5o semestres da área da saúde. Rev Nutr 2008; 21:39-47.

14. Evans AE, Sawyer-Morse MK, Betsinger A. Fruit and vegetable consumption among MexicanAmerican college students. J Am Diet Assoc 2000; 100:1399-402.

15. Vieira VCR, Priore SE, Ribeiro SMR, Franceschini SCC, Almeida LP. Perfil socioeconômico, nutricional e de saúde de adolescentes recém-ingressos em uma universidade pública brasileira. Rev Nutr 2002; 15:273-82.

16. Unusan N. Fruit and vegetable consumption among Turkish university students. Int J Vitam Nutr Res 2004; 74:341-8.

17. Adams T, Colner W. The association of multiple risk factors with fruit and vegetable intake among a nationwide sample of college students. J Am Coll Health 2008; 56:455-61.

18. Kish L. Survey sampling. New York: John Wiley and Sons; 1965.

19. Kalton G. Introduction to survey sampling. Beverly Hills: Sage; 1983.

20. Ainsworth BE, Haskell WL, Whitt MC, Irwin ML, Swartz AM, Strath SJ, et al. Compendium of physical activities: an update of activity codes and MET intensities. Med Sci Sports Exerc 2000; 32(9 Suppl): S498-504.

21. Haskell WL, Lee IM, Pate RR, Powell KE, Blair SN, Franklin BA, et al. Physical activity and public health: updated recommendation for adults from the American College of Sports Medicine and the American Heart Association. Circulation 2007; 116:1081-93.

22. Victora C, Huttly S, Fuchs S, Olinto M. The role of conceptual frameworks in epidemiological analysis: a hierarchical approach. Int J Epidemiol 1997; 26:224-7.

23. Barros AJD, Hirakata VN. Alternatives for logistic regression in cross-sectional studies: an empirical comparison of models that directly estimate the prevalence ratio. BMC Med Res Methodol 2003; 3:21.

24. Figueiredo ICR, Jaime PC, Monteiro CA. Fatores associados ao consumo de frutas, legumes e verduras em adultos da Cidade de São Paulo. Rev Saúde Pública 2008; 42:777-85.
25. Moura EC, Dias RM, Reis RC. Determinantes do consumo de frutas, legumes e verduras na população adulta de Belém, Pará, 2005. Nutrire Rev Soc Bras Aliment Nutr 2007; 32:29-40.

26. Neutzling MB, Rombaldi AJ, Azevedo MR, Hallal PC. Fatores associados ao consumo de frutas, legumes e verduras em adultos de uma cidade no Sul do Brasil. Cad Saúde Pública 2009; 25:2365-74.

27. Jaime PC, Figueiredo IC, Moura EC, Malta DC. Fatores associados ao consumo de frutas e hortaliças no Brasil, 2006. Rev Saúde Pública 2009; 43 Suppl 2:57-64.

28. Levy RB, Castro IRR, Cardoso LO, Tavares LF, Sardinha LMV, Gomes FS, et al. Consumo e comportamento alimentar entre adolescentes brasileiros: Pesquisa Nacional de Saúde do Escolar (PeNSE), 2009. Ciênc Saúde Coletiva 2010; 15 Suppl 2: S3085-97.

29. Darmon N, Ferguson EL, Briend A. A cost constraint alone has adverse effects on food selection and nutrient density: an analysis of human diets by linear programming. J Nutr 2002; 132:3764-71.

30. Claro RM, Monteiro CA. Renda familiar, preço de alimentos e aquisição domiciliar de frutas e hortaliças no Brasil. Rev Saúde Pública 2010; 44: 1014-20.

31. Kamphuis CB, Giskes K, Bruijn GJ, Wendel-Vos W, Brug J, van Lenthe FJ. Environmental determinants of fruit and vegetable consumption among adults-a systematic review. Br J Nutr 2006; 96: 620-35.

32. Seo DC, Nehl E, Agley J, Ma SM. Relations between physical activity and behavioral and perceptual correlates among midwestern college students. J Am Coll Health 2007; 56:187-97.

33. Keller S, Maddock JE, Hannover W. Thyrian JR, Basler H. Multiple health risk behaviors in German first year university students. Prev Med 2008; 46:189-95.

34. Dodd LJ, Al-Nakeeb Y, Nevill A, Forshaw MJ. Lifestyle risk factors of students: a cluster analytical approach. Prev Med 2010; 51:73-7.

35. Sebastian RS, Wilkinson Enns C, Goldman JD. US adolescents and MyPyramid: associations between fast-food consumption and lower likelihood of meeting recommendations. J Am Diet Assoc 2009; 109:226-35.

36. Drewnowski A, Popkin BM. The nutrition transition: new trends in the global diet. Nutr Rev 1997; 55:31-43.

37. Levy-Costa RB, Sichieri R, Pontes NS, Monteiro CA. Disponibilidade domiciliar de alimentos no Brasil: distribuição e evolução (1974-2003). Rev Saúde Pública 2005; 39:530-40.

38. Miller TM, Abdel-Msksoud MF, Crane LA, Marcus AC, Byers TE. Effects of social approval bias on self-reported fruit and vegetable consumption: a randomized controlled trial. Nutr J 2008; 7:18.

Recebido em 12/Set/2011

Versão final reapresentada em 02/Abr/2012

Aprovado em 17/Abr/2012 